\title{
A High Power Solar Electric Propulsion - Chemical Mission for Human Exploration of Mars
}

\author{
Laura M. Burke*, Michael C. Martini†, and Steven R. Oleson ${ }^{\ddagger}$
}

\begin{abstract}
Recently Solar Electric Propulsion (SEP) as a main propulsion system has been investigated as an option to support manned space missions to near-Earth destinations for the NASA Gateway spacecraft. High efficiency SEP systems are able to reduce the amount of propellant long duration chemical missions require, ultimately reducing the required mass delivered to Low Earth Orbit (LEO) by a launch vehicle. However, for long duration interplanetary Mars missions, using SEP as the sole propulsion source alone may not be feasible due to the long trip times to reach and insert into the destination orbit.

By combining an SEP propulsion system with a chemical propulsion system the mission is able to utilize the high-efficiency SEP for sustained vehicle acceleration and deceleration in heliocentric space and the chemical system for orbit insertion maneuvers and trans-earth injection, eliminating the need for long duration spirals. By capturing chemically instead of with low-thrust SEP, Mars stay time increases by nearly 200 days. Additionally, the size the of chemical propulsion system can be significantly reduced from that of a standard Mars mission because the SEP system greatly decreases the Mars arrival and departure hyperbolic excess velocities $\left(\mathrm{V}_{\infty}\right)$.
\end{abstract}

\section{Nomenclature}

$\begin{array}{ll}A C S & \text { Attitude Control System } \\ A T V & \text { Automated Transfer Vehicle } \\ C_{3} & \text { Characteristic energy, } \mathrm{km}^{2} / \mathrm{s}^{2} \\ D R A & \text { Design Reference Architecture } \\ D S H & \text { Deep Space Habitat } \\ E M-L 2 & \text { Earth-Moon L2 Lagarange Point } \\ E P & \text { Electric Propulsion } \\ g & \text { Earth standard gravity, } 9.80665 \mathrm{~m} / \mathrm{s}^{2} \\ H A B & \text { Habitat } \\ I M E M L 2 & \text { Initial Mass at EM-L2 } \\ I M L E O & \text { Initial Mass in Low Earth Orbit } \\ L E O & \text { Low Earth Orbit } \\ M O I & \text { Mars Orbit Insertion } \\ M P C V & \text { Multi-Purpose Crew Vehicle } \\ N A I F & \text { Navigation and Ancillary Information Facility } \\ N E P & \text { Nuclear Electric Propulsion } \\ N T R & \text { Nuclear Thermal Rocket } \\ R C S & \text { Reaction Control System } \\ S E P & \text { Solar Electric Propulsion } \\ S L S & \text { Space Launch System }\end{array}$

\footnotetext{
*Aerospace Engineer, Mission Design and Trajectory Analysis Branch, NASA Glenn Research Center, 21000 Brook Park Rd. Cleveland, OH 44135

$\dagger$ Aerospace Engineer, Mission Design and Trajectory Analysis Branch, Vantage Partners, 21000 Brook Park Rd. Cleveland, $\mathrm{OH} 44135$

${ }^{\ddagger}$ COMPASS Team Lead, Mission Design and Trajectory Analysis Branch, NASA Glenn Research Center, 21000 Brook Park Rd. Cleveland, OH 44135
} 
TMI Trans-Mars Injection

TOF Time of Flight

$V_{\infty} \quad$ Hyperbolic Excess Velocity, $\mathrm{km} / \mathrm{s}$

$\Delta V \quad$ (Delta-V) Imparted Velocity Change, $\mathrm{km} / \mathrm{s}$

\section{Introduction}

This analysis was performed to support a COMPASS Team ${ }^{\mathrm{a}}$ study that considered a 2037 conjunction class crewed Earth-Mars round trip mission utilizing a vehicle with an SEP system comprised of eight 125 $k W$ Hall thrusters and a chemical system with a $7000 \mathrm{lbf}$ Orion-derived bi-propellent thruster. The SEP Module will be injected into a $400 \mathrm{~km}$ LEO and spiral-out to Earth-Moon L2 (EM-L2) where it will rendezvous with the NASA Gateway spacecraft. The Multi-Purpose Crew Vehicle (MPCV) and crew will be on a separate launch direct to EM-L2 for rendezvous. The baseline mission for this study is to deliver six crew from an EM-L2 halo orbit to a 1-sol elliptical Mars orbit for a 300-day stay and return to direct re-entry at Earth by the crew capsule. Additionally, all-SEP mission options were investigated for comparison. Results of this study show that a mission with a combined SEP-Chemical system may be the lowest mass option currently available for a manned Mars long stay mission by requiring only two Space Launch System (SLS) Block-2 (SLS-2B) launches to deliver the SEP-Chemical vehicle to LEO.

Five different mission architectures were investigated during the COMPASS study. The focus of this paper will be on the trajectory design for the baseline mission as well as the all-SEP option. Due to the limited length of time of a COMPASS design session, the original analysis was more limited in the scope of the power and inert mass trades to only encompass parameters near the estimated vehicle design. The trajectory analysis was expanded for this paper to included a more in-depth look at power to the thrusters, SEP system specific impulse, and a wider range of spacecraft inert masses. Additionally, through this additional analysis it was found that by allowing the optimizer to insert a third SEP burn on the outbound leg that a slightly more optimal solution could be found than was used for the original COMPASS design which was constrained to use only two SEP burns during the outbound leg.

\section{Mission Overview}

\section{A. Ground Rules and Assumptions}

For this study SEP and Chemical propulsion systems have been combined (SEP-Chem) as an alternate approach to a nuclear bimodal architecture which utilize Nuclear Thermal Rockets (NTR) and Nuclear Electric Propulsion (NEP). The goal of this COMPASS study was to demonstrate that a SEP-Chem architecture utilizing no more than $1 M W$ was capable of performing a human Mars 300 day stay orbital mission within 1000 days total mission time. ${ }^{1}$ In order to make this mission architecture easily comparable to other piloted Mars architectures, one figure of merit for this study was the number of heavy lift SLS launches required to deliver all the vehicle elements into orbit. To minimize the number of SLS-2B launches, the SEP Module and $\mathrm{HAB}$ Module elements are designed to each fit within the volume and mass constraints of a single SLS-2B launch vehicle. In the event that the mass exceeds the limits allowable by the SLS-2B, the excess mass (allocated as Xenon propellant), will be delivered separately with an Automated Transfer Vehicle (ATV).

To maintain consistency with DRA $5.0{ }^{2}$ the 2037 launch opportunity was selected to represent the most stressing "hard" long-stay, conjunction class mission across the synodic cycle. Typical mission $\Delta V$ 's will be less for other, less stressing opportunities during this synodic cycle. In addition to the SEP stage, the system elements include a $24 t \mathrm{MPCV}$ and a $53 t$ Deep Space Habitat (DSH). The SEP Module and the HAB Module will be delivered via an SLS-2B launch into LEO at which point they will be autonomously assembled. Following assembly, the SEP system will be used to spiral the assembled vehicle up to an EM-L2

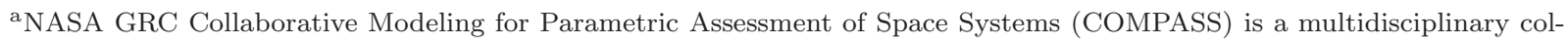
laborative engineering team whose primary purpose is to perform integrated vehicle systems analysis and provide conceptual designs and trades for both Exploration and Space Science Missions
} 
Halo orbit where the vehicle will station-keep until rendezvous with the MPCV. The MPCV carrying six crew members will be launched separately direct to EM-L2.

In order to determine the proper operating conditions for the SEP-Chem vehicle for this mission, sweeps on the SEP system performance were performed.

EP Propulsion System Performance Ranges:

- End of Life Power to SEP system $=800 \mathrm{~kW}$ to $1200 \mathrm{~kW}$

- Chemical system $I_{s p}$ for MOI and TEI $=327.5 \mathrm{~s}$

- Specific Impulse of SEP System: 2000-3000 $s$

- Total System Efficiency: $65 \%$

The SLS-2B is used to deliver the SEP Module and HAB Module into LEO. For the purposes of this study the following performance estimate was used:

- SLS-2B Launch capability of $123 t$ to a $-92.6 \mathrm{~km}$ x $407 \mathrm{~km}$ orbit $^{3}$

- Customer Reserve $(5 \%)=6.15 t$

- Spacecraft Adapter $(2.5 \%)=2.92 t$

- Net Payload to LEO $=113.93 t$

- Utilize appropriate kick stage to insert each element into $407 \mathrm{~km}$ circular orbit

- Perigee raise $\Delta V=148 \mathrm{~m} / \mathrm{s}$

For the COMPASS study baseline design, some propellants were divided between the SEP and HAB elements in the following manner to achieve the desired wet mass at launch for each element:

- 33,500 $\mathrm{kg}$ of chemical propellant was placed on the SEP Module, remaining chemical propellant was placed on the HAB Module

- The Xenon propellant load on the SEP Module was adjusted such that the Module had a mass of $113,755 \mathrm{~kg}$ on the launch pad (full SLS-2B launch payload)

- Remaining Xenon propellant was placed on the HAB Module

- Any amount that the HAB Module was over the allowed SLS-2B launch mass by was assumed to be taken up separately in ATV flights

As a starting point for analysis, the inert mass of the vehicle was estimated using assumptions based off of previous COMPASS studies. A sufficient range of vehicle inert masses to encompass both vehicle growth or reduction from this point was selected in order to also capture the actual vehicle mass of the COMPASS design.

- SEP Stage

- Power/propulsion subsystem $=19 \mathrm{~kg} / \mathrm{kW}$

- Xenon tanks $=4 \%$ of EP propellant

- Structure+Balance of Vehicle $=25 \%$ of vehicle dry mass

- Chemical Stage $=5000 \mathrm{~kg}$ fixed mass $+15 \%$ of the chemical propellant

- $\mathrm{DSH}=53 t$

- $\mathrm{MPCV}=24 t$

With these assumptions, the size of the SEP-Chem vehicle was estimated as $\sim 115 t$ for a $800 \mathrm{~kW}$ vehicle. The accuracy of the individual preliminary sizing equations was not assessed following the completion of the COMPASS design of the SEP-Chem vehicle, however, these equations do slightly underestimate the actual mass as the final design of the baseline $800 \mathrm{~kW}$ vehicle has an inert mass of $121 \mathrm{t}^{3}$ 


\section{B. Mission and Technology Trades}

The complete list of architecture options explored during the COMPASS study is listed in Table 1.

\begin{tabular}{|c|c|c|c|c|}
\hline Option & LEO to EM-L2 & $\begin{array}{c}\text { Earth Flyby on } \\
\text { Departure }\end{array}$ & $\begin{array}{c}\text { Interplanetary } \\
\text { Propulsion }\end{array}$ & $\begin{array}{l}\text { MOI/TEI } \\
\text { Propulsion }\end{array}$ \\
\hline 1 (SEP Tug) & SEP & Chem/SEP & None & Chem \\
\hline 2 & SEP & Chem/SEP & SEP & Chem \\
\hline 3 (Baseline) & SEP & None & SEP & Chem \\
\hline 4 (All-SEP) & SEP & None & SEP & SEP \\
\hline 5 & SEP & Chem/SEP & SEP & SEP \\
\hline 6 (Cargo) & SEP & None & SEP & Aerocapture \\
\hline
\end{tabular}

Figure 1. Mission Architecture Options for COMPASS Study

The main focus of this paper will be on Options 3 and 4. The combined SEP-Chem vehicle, Option 3, was determined to be the best performing architecture option for this mission. More detailed information on the other options as well as a complete description of the vehicle conceptual design can be found in References 1 and 4 .

Technology trades for the primary propulsion system, capture propulsion system, power level, and the specific impulse of the SEP system were performed for this study. The specific ranges these trades covered are listed below:

- Primary Propulsion System: All SEP or SEP+Chemical

- Propulsion System for Mars Capture and Departure: Chemical or SEP

- Power to SEP System: 800-1200 kW

- Specific Impulse of SEP System: 2000-3000 $s$

\section{Analysis Tools}

\section{A. COPERNICUS}

COPERNICUS was used to model the Earth-Mars SEP-Chem trajectories and perform the analysis presented in this paper. COPERNICUS is a 3-Degrees of Freedom spacecraft trajectory design and optimization program that was originally developed at the University of Texas with recent developments and maintenance being done by Johnson Space Center. ${ }^{5}$ Trajectory models are built as a series of segments propagated in either a point-mass or higher order gravitational field. The segments can be connected and constrained with the addition of linear or non-linear constraints. COPERNICUS has the capability to model both low thrust burns as well as high thrust burns as either impulsively or with finite maneuvers for propulsion systems which can be based on chemical, solar electric, or nuclear powered engines. SPICE files provided by Jet Propulsion Laboratory's NAIF is the basis of coordinate systems and ephemeris data.

\section{B. MALTO}

The Mission Analysis Low-Thrust Optimization program (MALTO) is a Jet Propulsion Laboratory developed preliminary mission design tool for low-thrust applications in which a user formulates trajectories by dividing the end-to-end mission into legs which begin and end at control points. It is low-fidelity in the sense that low-thrust arcs are approximated by a series of impulsive maneuvers and the only gravitational force modeled is that of the central body. MALTO can model solar-electric propulsion (SEP), nuclear-electric propulsion (NEP), and solar-sail trajectories. ${ }^{6}$ Ephemeris data is provided by SPICE files produced by Jet Propulsion Laboratory's NAIF. 


\section{Baseline SEP-Chem Vehicle Description}

Once assembled, the integrated SEP-Chem vehicle consists of an SEP Module, a HAB Module, and a $24 t \mathrm{MPCV}$. The mass estimate for the MPCV is based on recent Gateway mission analysis. ${ }^{7}$ The total inert mass for the fully assembled baseline SEP-Chem vehicle with the fully-loaded MPCV is 121t. A graphic showing the fully assembled vehicle with the SEP and HAB Modules with the main components identified is shown in Figure 2. The internal components of the SEP-Chem vehicle including the relative sizes of the Xenon and chemical propellant tanks and the EP and Orion derived chemical thrusters is shown in Figure 3.

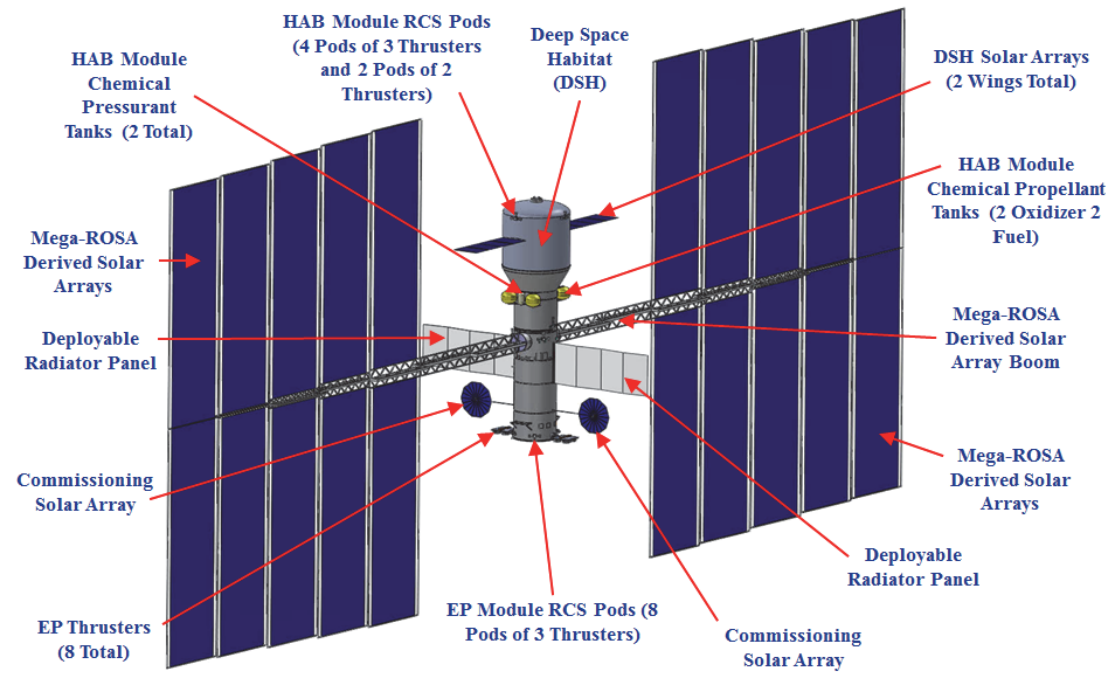

Figure 2. Concept Design and Key Components of the SEP and HAB Modules following Assembly in Low Earth Orbit

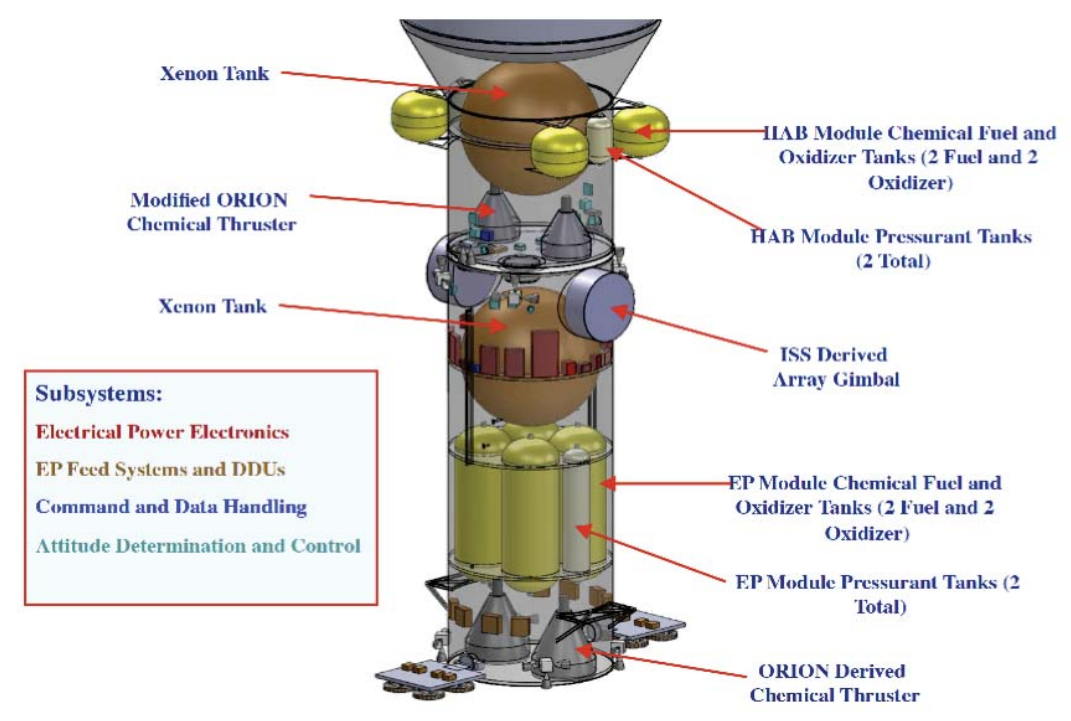

Figure 3. Internal Components of the SEP-Chem Vehicle

Additional top-level details of the SEP Module and HAB Module are discussed within the subsequent subsections of this document. More detailed information about the COMPASS design of the SEP and HAB Modules can be found in References 1 and 4 . 


\section{A. SEP Module}

The SEP propulsion system is made up of eight $125 \mathrm{~kW}$ nested Hall thrusters, (six primary and two spares) operating at $2400 \mathrm{~s}$ with $65 \%$ efficiency. Power to the thrusters is supplied by a $500 \mathrm{~V}$ Mega ROSA array sized to provide $800 \mathrm{~kW}$ EOL power at $1 \mathrm{AU}$. The main solar array is made up of two $2383 \mathrm{~m}^{2}$ wings, one on each side of the vehicle, populated with $33 \%$ efficient IMM solar cells.

Four of the Hall thrusters are gimbaled to augment the vehicle's ACS, nominally provided by the RCS thrusters, with the remaining four being statically mounted. Each Hall thruster has a corresponding directdrive unit mounted near the thrusters. Xenon was chosen as the propellant because its low ionization energy enables high thruster efficiency and is more easily stored than other heavy noble gases. Two $3.9 \mathrm{~m}$ diameter spherical composite overwrap pressure vessel tanks store the Xenon as a supercritical gas at 1200 psia. The chemical propulsion system consists of two Orion derived bipropellant chemical engines mounted on the bottom deck of the vehicle with the propellant tanks mounted directly above them.

A mass breakdown of the total vehicle mass at launch is shown in Table 4. The total wet mass with growth of the SEP Module was constrained to not exceed the capability of a single SLS-2B launch vehicle, $113.8 t .^{3}$

\begin{tabular}{|c|c|c|c|c|}
\hline SEP Piloted SLS Launch 2 - SEP Module & 107397 & 3408 & 110805 & $3 \%$ \\
\hline Science & 0 & 0 & 0 & TBD \\
\hline Attitude Determination and Control & 45 & 1 & 47 & $3 \%$ \\
\hline Command and Data Handling & 121 & 23 & 144 & $19 \%$ \\
\hline Communications and Tracking & 55 & 15 & 70 & $28 \%$ \\
\hline Electrical Power Subsystem & 6915 & 1110 & 8025 & $16 \%$ \\
\hline Thermal Control (Non-Propellant) & 2506 & 451 & 2957 & $18 \%$ \\
\hline Propulsion (Chemical Hardware) & 2310 & 375 & 2685 & $16 \%$ \\
\hline Propellant (Chemical) & 34269 & & 34269 & owe \\
\hline Propulsion EP Hardware) & 3680 & 437 & 4117 & $12 \%$ \\
\hline Propellant (EP) & 51870 & & 51870 & oflu \\
\hline Structures and Mechanisms & 5626 & 994 & 6620 & $18 \%$ \\
\hline \multirow{3}{*}{$\begin{array}{l}\text { Element } 2 \text { consumables (if used) } \\
\text { Estimated Spacecraft Dry Mass } \\
\text { Estimated Spacecraft Wet Mass }\end{array}$} & 0 & & 0 & \multirow{3}{*}{$16 \%$} \\
\hline & 21258 & 3408 & 24666 & \\
\hline & 107397 & 3408 & 110805 & \\
\hline \multicolumn{4}{|l|}{ - Growth Calculations SEP Piloted SLS Launch 2 - S } & Total Growth \\
\hline Dry Mass Desired System Level Growth & 21258 & 6377 & 27636 & $30 \%$ \\
\hline Additional Growth (carried at system level) & & 2969 & & $14 \%$ \\
\hline Total Wet Mass with Growth & 107397 & 6377 & 113775 & \\
\hline
\end{tabular}

Figure 4. Mass Breakdown of COMPASS Designed SEP Module

\section{B. HAB Module}

The DSH within the HAB Module is sized based on the number of crew and mission duration with mass estimates resulting from parametric sizing performed by the HAT DSH team. ${ }^{8}$ This design study assumes a crew of 6 and has a maximum mission duration goal of 1000 days resulting in a DSH and system mass of $53.68 t$.

In addition to the dry mass of the DSH and supporting structure, the HAB Module also carries the necessary components of a chemical propulsion system in order to perform the perigee raise maneuver following insertion into orbit via the SLS-2B. Since the total wet mass of the SEP Module was constrained to be no more than a single SLS-2B can deliver to orbit, any additional propellant is carried in tanks located within the HAB module. The total wet mass of the HAB Module is 128.4t. Table 5 provides a complete mass breakdown of the HAB Module at launch. Since the mass of this component exceeds the SLS-2B capability, two ATV class tanker flights, capable of delivering $\sim 8 \mathrm{t}$ to orbit, will be used to carry the extra Xenon propellant. ${ }^{3}$ 


\begin{tabular}{|c|c|c|c|c|}
\hline Main Subsystems & $\begin{array}{c}\text { Basic Mass } \\
(\mathrm{kg})\end{array}$ & $\begin{array}{l}\text { Growth } \\
\text { (kg) }\end{array}$ & $\begin{array}{l}\text { Predicted } \\
\text { Mass (kg) }\end{array}$ & \begin{tabular}{|c|} 
Aggregate \\
Growth (\%)
\end{tabular} \\
\hline SEP Mars Piloted Vehicle & 234058 & 4256 & 238313 & \\
\hline SEP Piloted SLS Launch 1- HAB Module & 126660 & 848 & 127508 & $1 \%$ \\
\hline Habitat and systems & 53680 & 0 & 53680 & $0 \%$ \\
\hline Attitude Determination and Control & 92 & 3 & 95 & $3 \%$ \\
\hline Command and Data Handling & 47 & 10 & 57 & $21 \%$ \\
\hline Communications and Tracking & 0 & 0 & 0 & TBD \\
\hline Electrical Power Subsystem & 46 & 23 & 69 & $50 \%$ \\
\hline Thermal Control (Non-Propellant) & 398 & 72 & 470 & $18 \%$ \\
\hline Propulsion (Chemical Hardware) & 1179 & 155 & 1334 & $13 \%$ \\
\hline Propellant (Chemical) & 9644 & & 9644 & (5) \\
\hline Propulsion (EP Hardware) & 1509 & 127 & 1636 & $8 \%$ \\
\hline Propellant (EP) & 57359 & & 57359 & Tow \\
\hline Structures and Mechanisms & 2706 & 458 & 3164 & $17 \%$ \\
\hline Element 1 consumables (if used) & 13992 & & 13992 & \\
\hline Estimated Spacecraft Dry Mass (no prop,consum) & 45665 & 848 & 46513 & $2 \%$ \\
\hline Estimated Spacecraft Wet Mass & 126660 & 848 & 127508 & \\
\hline \multicolumn{4}{|c|}{ Growth Calculations SEP Piloted SLS Launch 1 - HAB Module } & Total Growth \\
\hline Dry Mass Desired System Level Growth & 5977 & 1793 & 7771 & $30 \%$ \\
\hline Additional Growth (carried at system level) & & 946 & & $16 \%$ \\
\hline Total Wet Mass with Growth & 126660 & 1793 & 128454 & \\
\hline
\end{tabular}

Figure 5. Mass Breakdown of COMPASS Designed HAB Module

\section{Trajectory Analysis}

\section{A. Combined SEP-Chemical Trajectories}

The SEP-Chem vehicle will be autonomously assembled in LEO after which it will use the SEP system to spiral to a Halo orbit around EM-L2. Spiral to the EM-L2 Halo requires $6650 \mathrm{~m} / \mathrm{s}$ of $\Delta V$ and takes 400 days for an $800 \mathrm{~kW}$ vehicle. ${ }^{3}$ The crew and MPCV will launch directly to EM-L2 from a SLS Block-1 (SLS-1B) on a four day trajectory to rendezvous with the SEP-Chem vehicle and depart to Mars.

By combining SEP with a small Orion-derived chemical system the benefits of each can be had by using each propulsion system during the appropriate phase of the mission. The SEP system can provide efficient, sustained acceleration in heliocentric space and by using the chemical system for capture and departure, the normally long spiral phases while in the Mars gravity well can be eliminated. Consequently, the Mars stay time in the destination orbit isn't reduced by the amount of time that an all-SEP vehicle requires to spiral in and out ( 190 days). The chemical system can be kept relatively small since the arrival and departure $V_{\infty}$ at Mars is greatly reduced by using the SEP system during interplanetary transit. Essentially the SEP system is used to nearly capture or escape the vehicle from Mars orbit, resulting in the eccentricity $=\sim 1$ just prior to the Mars Orbit Insertion (MOI) burn and just following the trans-Earth Injection (TEI) burn with the chemical system performing a final maneuver to insert or depart from the destination orbit.

The trajectory analysis for the SEP-Chem missions was performed using COPERNICUS. The starting location of the vehicle within COPERNICUS is a $30,000 \mathrm{~km}$ Az halo orbit at EM-L2. Due to the instability of Halo orbits around EM-L2, a $\Delta V$ is not required to depart the Halo since the vehicle will "fall-out" of the orbit after a relatively short period of no-orbital maintenance. Following EM-L2 departure, the vehicle coasts for a short period of time (never exceeding 2-weeks) prior to initiating the first of three outbound SEP burns. This initial SEP burn is relatively short compared to subsequent burns, lasting between 0 and 13 days varying mostly with inert mass and SEP $I_{s p}$. A coast lasting up to 110 days separates the first and second SEP burns. The second SEP burn varies in length between 75 days up to over 300 days in near all-propulsive outbound-leg scenarios. These first two burns essentially provide the positive $C_{3}$ that would normally be supplied by a chemical trans-Mars Injection (TMI) stage. An additional coast separates this burn from the final outbound SEP burn. This SEP burn occurs just prior to MOI and is used to reduce the Mars arrival $V_{\infty}$ which in turn reduces the amount of $\Delta V$ necessary for the chemical propulsion system to capture the vehicle into a 1-sol Mars orbit. MOI and TEI are performed by an Orion-derived chemical propulsion system. Stay time at Mars is constrained to be 300 days. Immediately after TEI the fourth SEP burn is performed followed by a long coast back to Earth. Although a fifth SEP burn was allowed close to Earth arrival to reduce the incoming $V_{\infty}$ it was eliminated by the optimizer. At Earth the MPCV separates from the SEP-Chem vehicle and performs a direct re-entry. The velocity at Earth arrival was constrained to be no more than $11.5 \mathrm{~km} / \mathrm{s}$. Including the 300 day stay at Mars, the total crewed time of the mission is approximately 1050 days which is $\sim 115-150$ days longer than the total crewed time of an NTP or all-chemical 
conjunction-class mission. This longer mission time is off-set by the reduction in SLS-2B launches ( 3-7 down to 2).

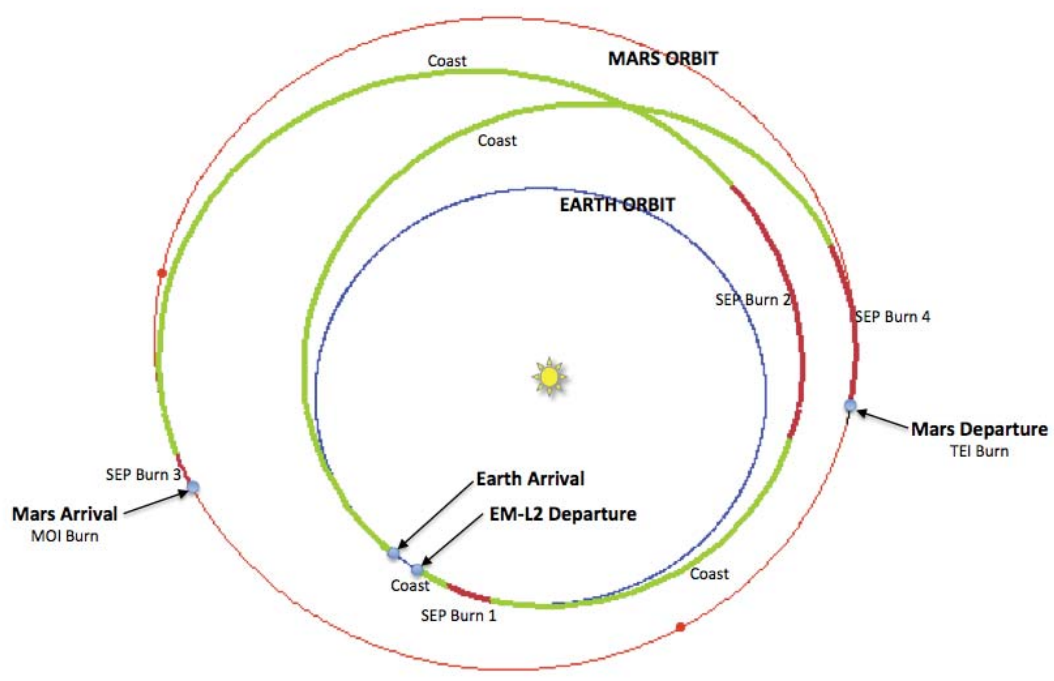

Figure 6. Ecliptic View of 2037 Reference SEP-Chem Roundtrip Mission with 300d Mars Stay

An ecliptic view of a 2037 SEP-Chem trajectory is showing in Figure 6. The green segments represent coasting phases while the red segments represent SEP burns. The trajectory pictured represents a higher power, lower $I_{s p}$ propulsion system. Since the duration of SEP Burn 1 and the coast separating SEP Burn 1 and SEP Burn 2 are not constrained to a minimum value, eventually they are eliminated by the optimizer as mass of the vehicle and the SEP $I_{s p}$ increase. In these cases coasting on the outbound leg is reduced substantially in order to provide more time to perform the outbound SEP burns, eventually leading to a near all-propulsive outbound leg.

\section{RESULTS OF BASELINE TRAJECTORY ANALYSIS}

The optimal stay time at Mars for an $800 \mathrm{~kW}, 125 t$ (slightly larger than the COMPASS baseline vehicle discussed in Section B) vehicle ranges from 258 to 172 days depending on the inert mass and SEP $I_{s p}$ as shown in Figure 7. The optimal stay time never reaches 300 days for power levels between $800 \mathrm{~kW}$ and $1200 \mathrm{~kW}$, therefore, a constraint needs to be implemented to achieve the minimum stay time of 300 days. Figure 8 shows the variations in initial mass at the EM-L2 (IMEML2) point for the various power levels when operating at different SEP specific impulses. IMEML2 represents the mass of the entire vehicle after it has rendezvous with the MPCV and crew. Figure 8(a) shows for a $125 t$ inert mass what the initial mass at EM-L2 would be if no minimum stay time is required. Figure 8(b) shows the same if stay time is constrained to be 300 days. When compared to the overall vehicle mass, the difference between initial mass in these two scenarios is not substantial (on the order on tons). However, when stay time is increased to 300 days it does becomes harder for lower power vehicles to accomplish the mission. For each power level there is a maximum SEP $I_{s p}$ after which it is no longer possible to complete the mission with the given constraints. This occurs because the limited thrust makes it such that the SEP propulsion system cannot physically push the vehicle mass quick enough between Earth and Mars within the allowable interplanetary transit time. As a consequence of the stay time constraint, the $I_{s p}$ of the SEP system capable of performing this mission is reduced from $2800 s$ down to $2700 s$. For the COMPASS design, the lowest power level and $I_{s p}$ (with some margin for increasing inert mass) that could accomplish a 300 day stay mission for an anticipated vehicle inert mass of near $115 t$ was selected as the baseline mission option. By baselining a lower power system, 800 $k W$, the mass of the propulsion system and solar array area required for the vehicle is reduced. 


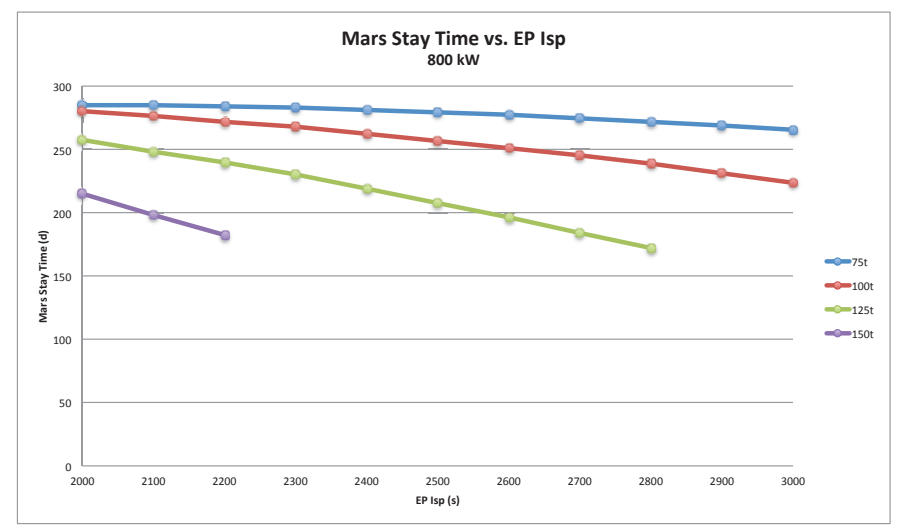

Figure 7. Stay Time vs. SEP Isp, Power $=800 \mathrm{~kW}$

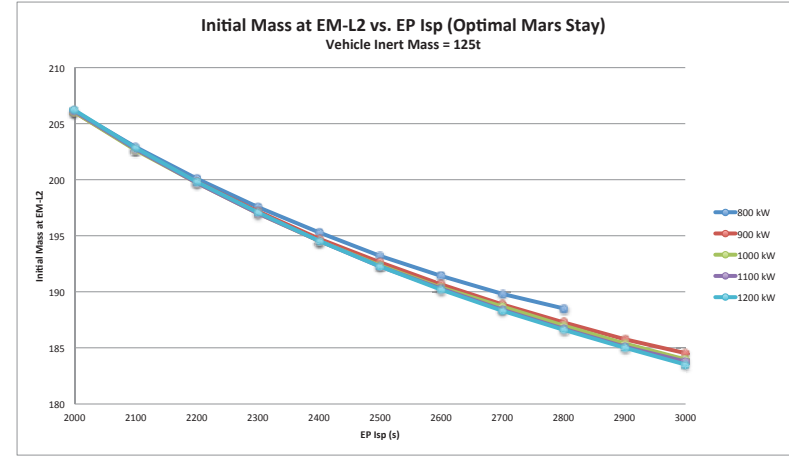

(a) Optimal Stay

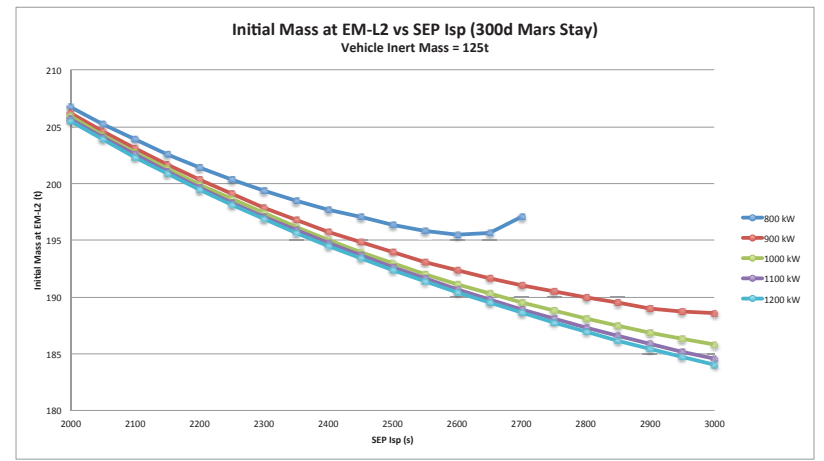

(b) $300 \mathrm{~d}$

Figure 8. Initial Mass at EM-L2 vs. SEP Isp for Optimal and Constrained Mars Stay Times

To compare the launch mass of the vehicle to the launch capability of two SLS-2B launch vehicles, the initial mass in LEO (IMLEO) was calculated based on a spiral-out $\Delta V$ of $6650 \mathrm{~m} / \mathrm{s}$. IMLEO represents the total launch mass of the SEP and HAB Modules that the SLS-2B will be required to deliver to LEO. IMLEO does not include the mass of the MPCV and crew since they will be delivered separately by a SLS-1B directly to EM-L2. Figure 9 shows the total IMLEO of the vehicle as well as the IMEML2 following spiral-out and after rendezvous with the MPCV and crew. 


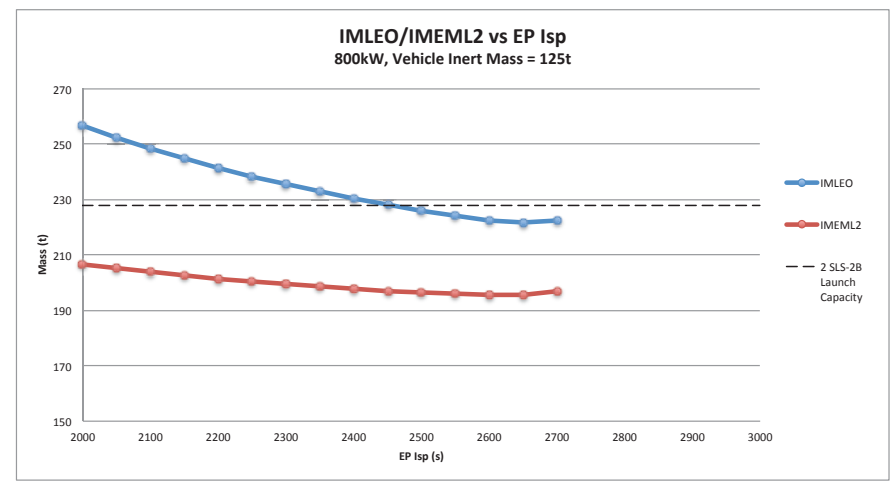

Figure 9. Mass vs. SEP Isp, Power $=800 \mathrm{~kW}$, Inert Mass $=125 \mathrm{t}$

Figure 9 shows that for an $800 \mathrm{~kW} 125 t$ inert vehicle operating at $\sim 2450 \mathrm{~s}$ that the total launch mass of the two vehicle elements fits within the capabilities of two SLS-2B launches. The baseline COMPASS design elected to set the $I_{s p}$ at $2400 s$. This $I_{s p}$ allowed for potential growth of the vehicle beyond $125 t$ as well as nearly fitting within the two SLS-2B launch goal. By selecting a higher $I_{s p}$ for a given power level the duration of the spiral to EM-L2 will increase, however, since this portion of the mission is completed autonomously the additional time does not impact crew time in space.

The following sets of plots show the detailed $\Delta V$ values required of the SEP and chemical systems to perform the mission for a given power, $I_{s p}$, and vehicle inert mass. Visual examination of the $\Delta V$ plots reveals that for lower mass vehicles $(75-100 t)$ increasing power, especially at lower $I_{s p}$ 's, does not have a significant effect on the SEP or chemical $\Delta V \mathrm{~s}$. When inert mass raises beyond this point increasing power becomes much more beneficial, enabling the SEP system to run at higher $I_{s p}$ 's and keeping MOI and TEI $\Delta V \mathrm{~s}$ low when compared to the lower power solutions. Also notable is that, in general, the outbound and inbound SEP $\Delta V$ s increase as SEP $I_{s p}$ increases while MOI and TEI $\Delta V$ s decrease as SEP $I_{s p}$ increases resulting in an overall lower IMLEO. 


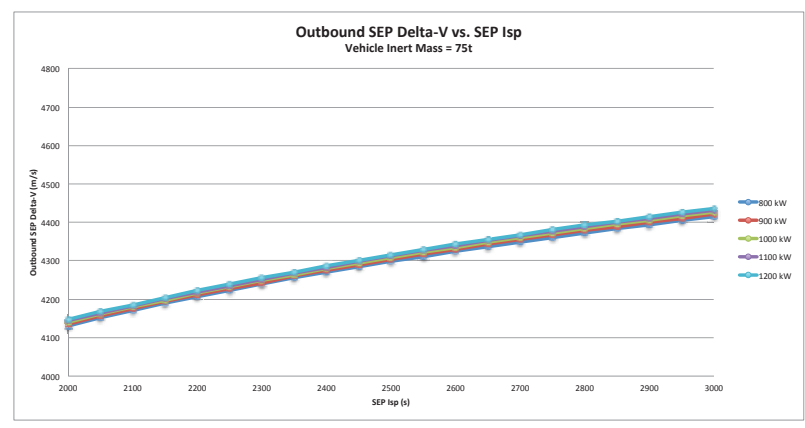

(a) $75 \mathrm{t}$

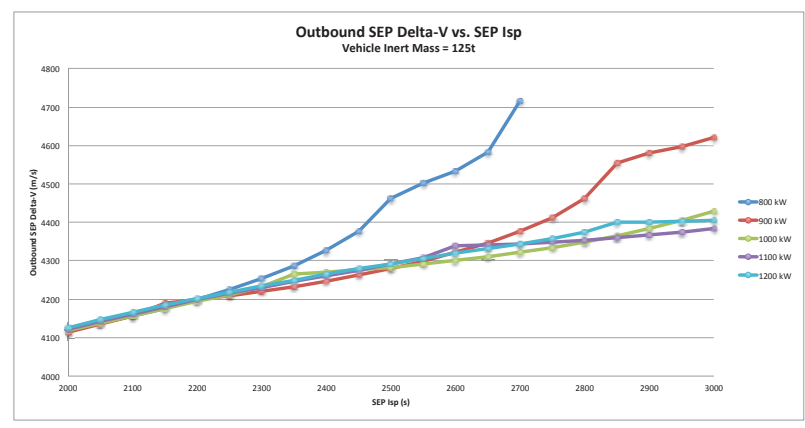

(c) $125 \mathrm{t}$

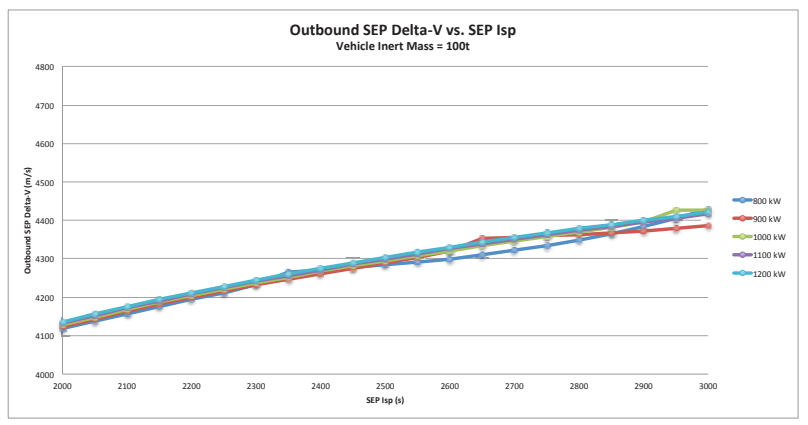

(b) $100 \mathrm{t}$

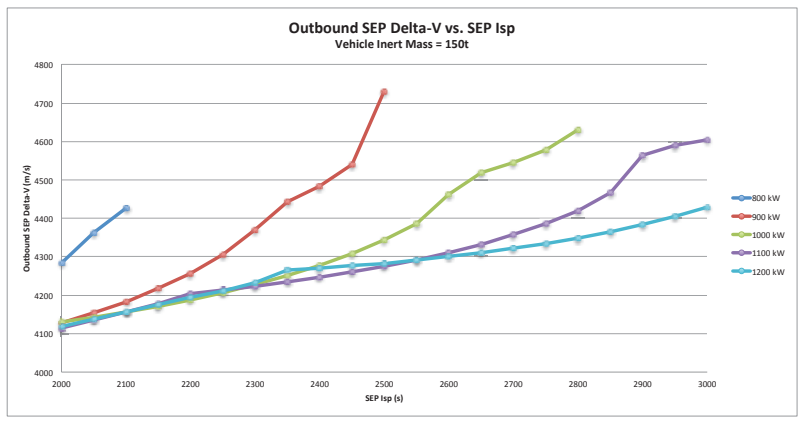

(d) $150 \mathrm{t}$

Figure 10. Outbound SEP Delta-V vs. SEP Isp

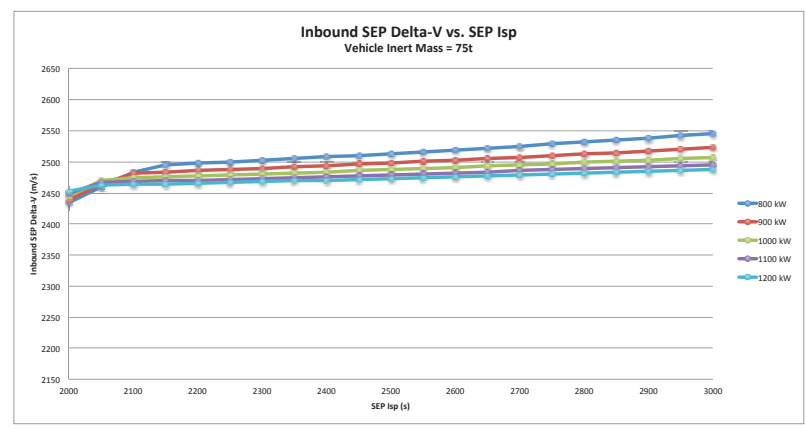

(a) $75 \mathrm{t}$

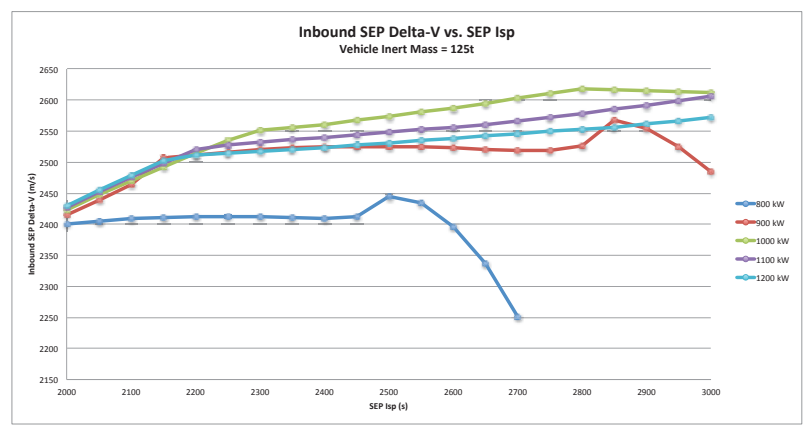

(c) $125 \mathrm{t}$

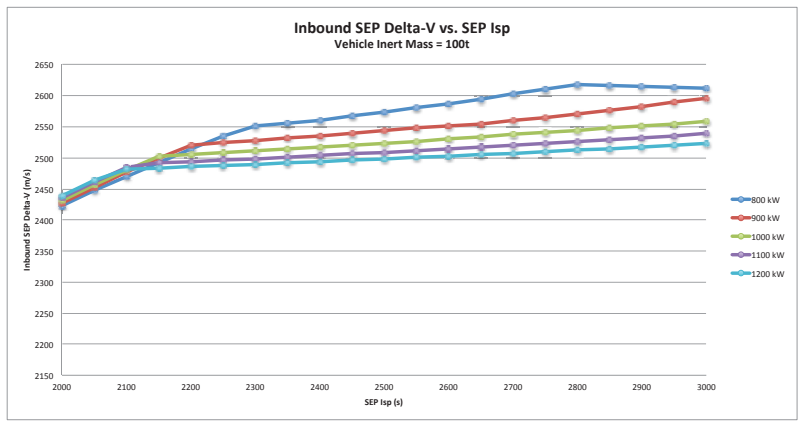

(b) $100 \mathrm{t}$

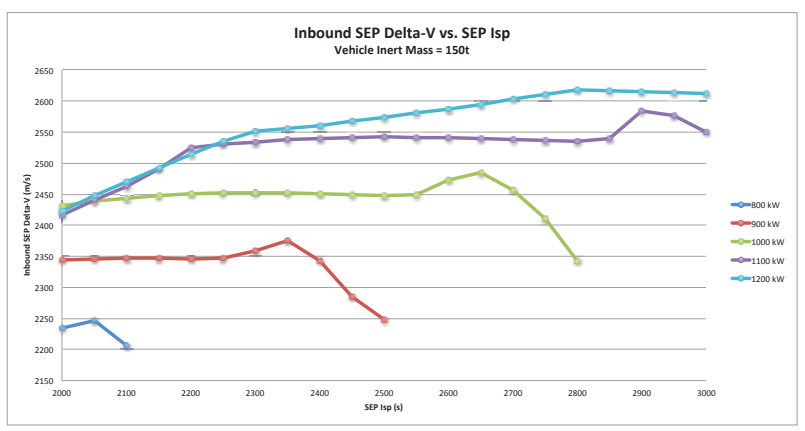

(d) $150 \mathrm{t}$

Figure 11. Inbound SEP Delta-V vs. SEP Isp

Figure 12 and Figure 13 show MOI and TEI $\Delta V$ vs SEP $I_{s p}$ for different vehicle inert masses respectively. MOI and TEI both decrease with increasing power. This is because with the higher power systems more of 
the overall required mission $\Delta V$ can be supplied by the SEP system since the same $\Delta V$ can be performed in a quicker manner than it could be with a lower power system. Hence, less energy needs to be taken out or imparted by the chemical system during Mars capture and departure. In some instances, the TEI $\Delta V \mathrm{~s}$ is reduced to a minimum of $\sim 227 \mathrm{~m} / \mathrm{s}$, supplying just enough energy to escape the 1-sol Mars orbit. This is the case for nearly all power levels and $I_{s p}$ 's for a $75 t$ inert vehicle in Figure 13(a).

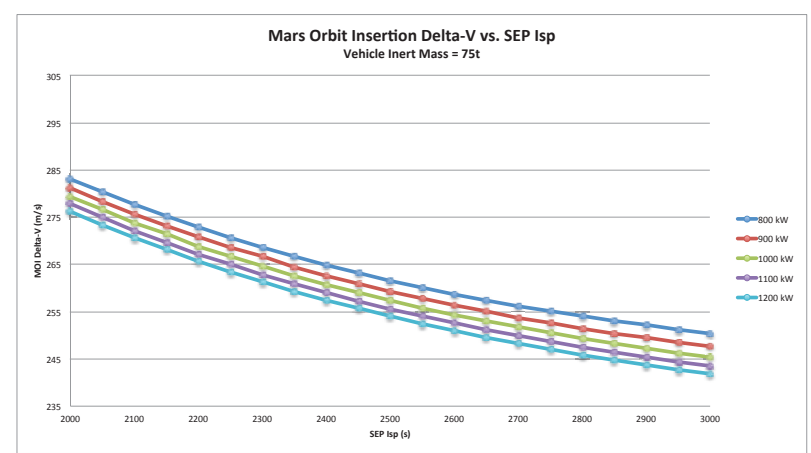

(a) $75 \mathrm{t}$

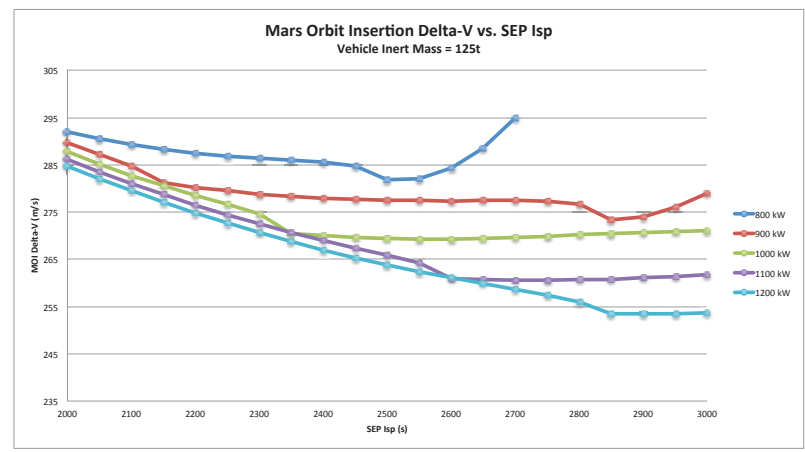

(c) $125 \mathrm{t}$

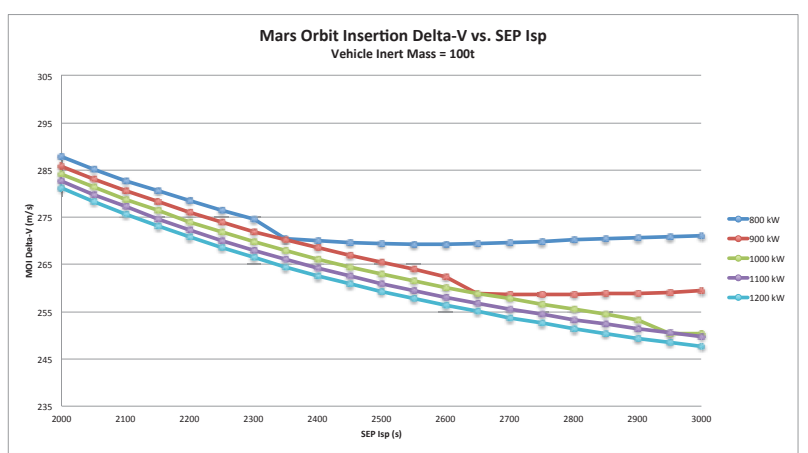

(b) $100 \mathrm{t}$

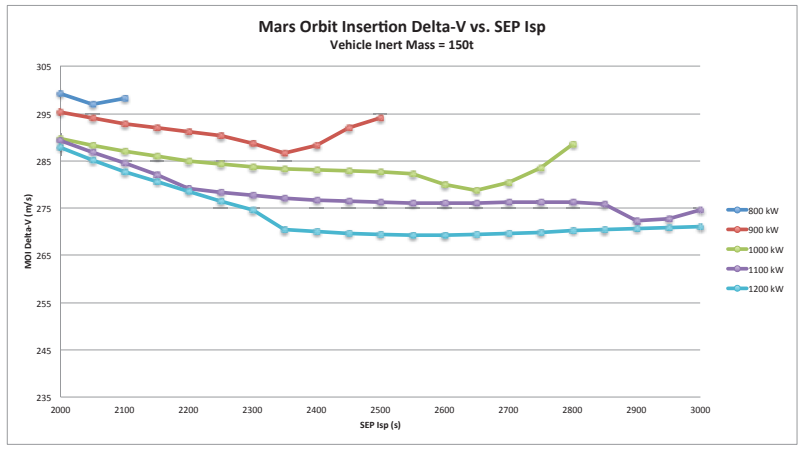

(d) $150 \mathrm{t}$

Figure 12. MOI Delta-V vs. SEP Isp 


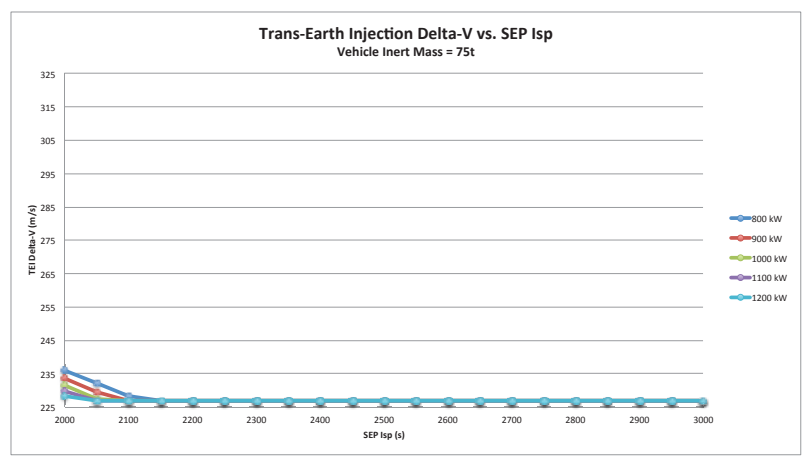

(a) $75 \mathrm{t}$

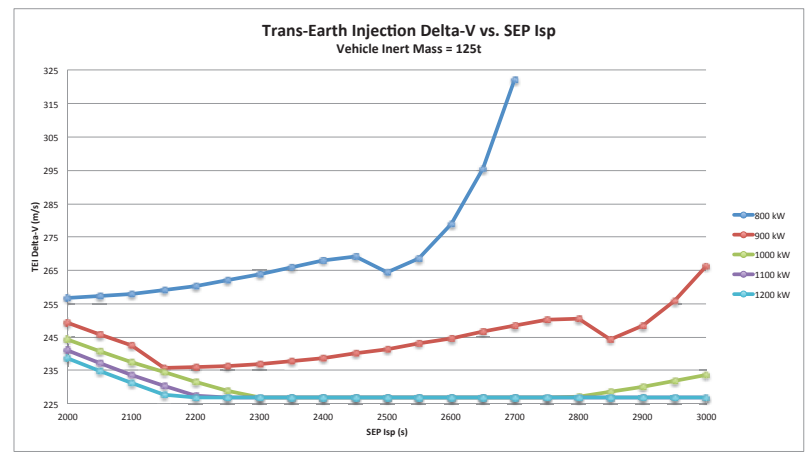

(c) $125 \mathrm{t}$

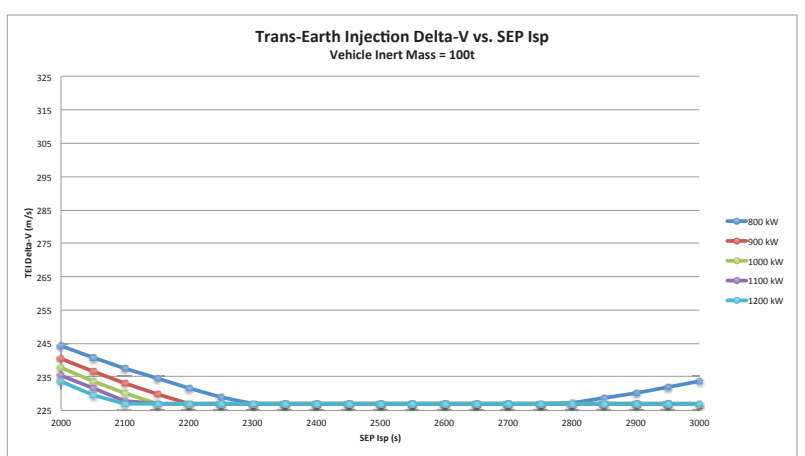

(b) $100 t$

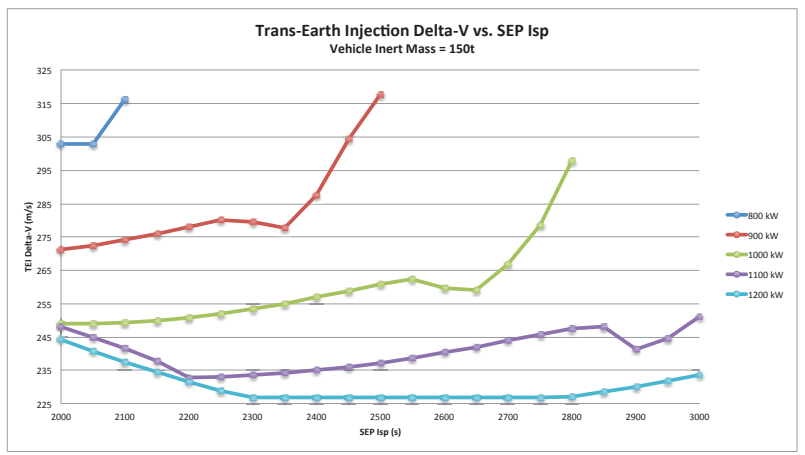

(d) $150 \mathrm{t}$

Figure 13. TEI Delta-V vs. SEP Isp

As expected, as the thrust-to-weight ratio of the vehicle decreases (lower power, higher $I_{s p}$, larger inert mass), the effectiveness of the SEP system to move the vehicle during the transit time decreases and the high-thrust chemical engines must be utilized to a greater degree in order to accomplish the mission within the mission timeframe while also maintaining a 300 day minimum Mars stay time.

The abrupt peaks and valleys in the above $\Delta V$ plots occur at the point when outbound Time of Flight (TOF) is at a minimum and inbound TOF is at a maximum for a given power level. Although there is not significant variation in the total mission TOF prior to this point, a slight dip in overall mission TOF occurs at this $I_{s p}$ as well. At $I_{s p}$ 's above this, the total mission TOF begins to rapidly increases along with the outbound SEP, MOI, and TEI $\Delta V$ s while inbound SEP $\Delta V$ rapidly decreases. For a $125 t, 800$ $k W$ vehicle this transition $I_{s p}$ is approximately 2500s. From Figure 8, 2500s is very near to the optimal $I_{s p}$ of $2600 \mathrm{~s}$ to minimize IMEML2 for a $800 \mathrm{~kW}$ 125t vehicle. For $I_{s p}$ 's not significantly higher than the optimal $I_{s p}$, the SEP-Chem trajectories become infeasible, unable to meet mission time constraints while maintaining mass and time continuity between COPERNICUS trajectory segments. Any noticeable bend in the trajectory prior to these easily identified transition points can be attributed to the location at which SEP Burn 1 is eliminate from the outbound leg of the trajectory (as seen at $2200 \mathrm{~s}$ for a $1100 \mathrm{~kW}, 150 t$ vehicle in Figure 10(d)).

From the COMPASS study, the total inert mass for the fully assembled baseline SEP-Chem vehicle with the fully-loaded MPCV is 121t. Table 14 shows a breakdown of the SEP and chemical $\Delta V$ s for a vehicle with an inert mass of 121t. By allowing an additional outbound leg SEP burn in this current analysis, the $\Delta V \mathrm{~s}$ are slightly improved over the original study $\Delta V \mathrm{~s}$ which only used two SEP burns during EM-L2 to Mars transit. 


\begin{tabular}{|l|c|}
\hline & Delta-V (m/s) \\
\hline Outbound EP & 4317 \\
\hline MOI & 283 \\
\hline TEI & 261 \\
\hline Inbound EP & 2433 \\
\hline
\end{tabular}

Figure 14. Representative Mission Delta-Vs for a 121t, 800kW, 2400s Vehicle

\section{B. All-SEP Trajectory}

For the all SEP trajectory option all propulsive maneuvers are performed with the SEP system. This includes capturing in to and out of Mars orbit, where it is assumed that the vehicle spirals down to and up from a 24-hr circular orbit using the SEP system. Trajectories were generated with MALTO using various combinations of power, $I_{s p}$ and Mars stay time, by maximizing the final mass returned to Earth for a given Mars stay time while constraining the total mission time to no longer than 1050 days. Obtained from each MALTO run was the maximum mass delivered back to Earth and the resulting required initial mass departing Earth's sphere of influence for a given power level, $I_{s p}$, and Mars stay time.

Like the SEP-Chem vehicle, the all-SEP will be autonomously assembled in LEO after which it will use the SEP system to spiral from LEO to a Halo orbit around EM-L2. Spiral to the EM-L2 halo requires 6650 $\mathrm{m} / \mathrm{s}$ of $\Delta V$ and 400 days for an $800 \mathrm{~kW}$ vehicle. The crew will launch directly to EM-L2 to rendezvous with the all-SEP vehicle and depart to Mars.

\section{RESULTS OF ALL-SEP TRAJECTORY ANALYSIS}

In order to perform a direct comparison to the baseline SEP-Chem COMPASS design, an all-SEP trajectory utilizing the same power and $I_{s p}$ was run to determine the maximum allowable inert mass and the corresponding IMLEO to achieve a given Mars stay time. Figure 15 shows the IMLEO and the maximum vehicle inert mass vs. Mars stay time for the equivalent all-SEP vehicle.

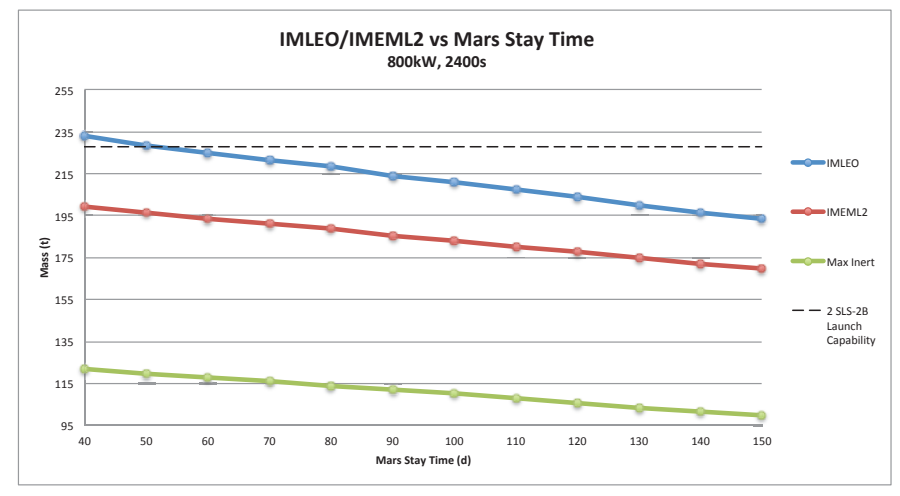

Figure 15. Mass vs. Stay Time for All-SEP Mission

Using the same power level and $I_{s p}(800 \mathrm{~kW}$ and 2400s) as the baseline SEP-Chem case, the resulting stay time at Mars for approximately the same size vehicle $(125 t)$ is about 40 days. The resulting required mass in LEO is $232 t$, which requires slightly more than two SLS-2B launches. The excess mass can be delivered in this case with two ATV flights. Although the IMLEO for this option is nearly equal to the baseline SEP-Chem vehicle, the stay time has been reduced from 300 days to 40 days. Not only does this significantly reduce the available science time at Mars but also exposes the crew for an additional 260 days to galactic cosmic radiation while in a microgravity environment. A plot of the interplanetary portion of this trajectory can be seen in Figure 16. 


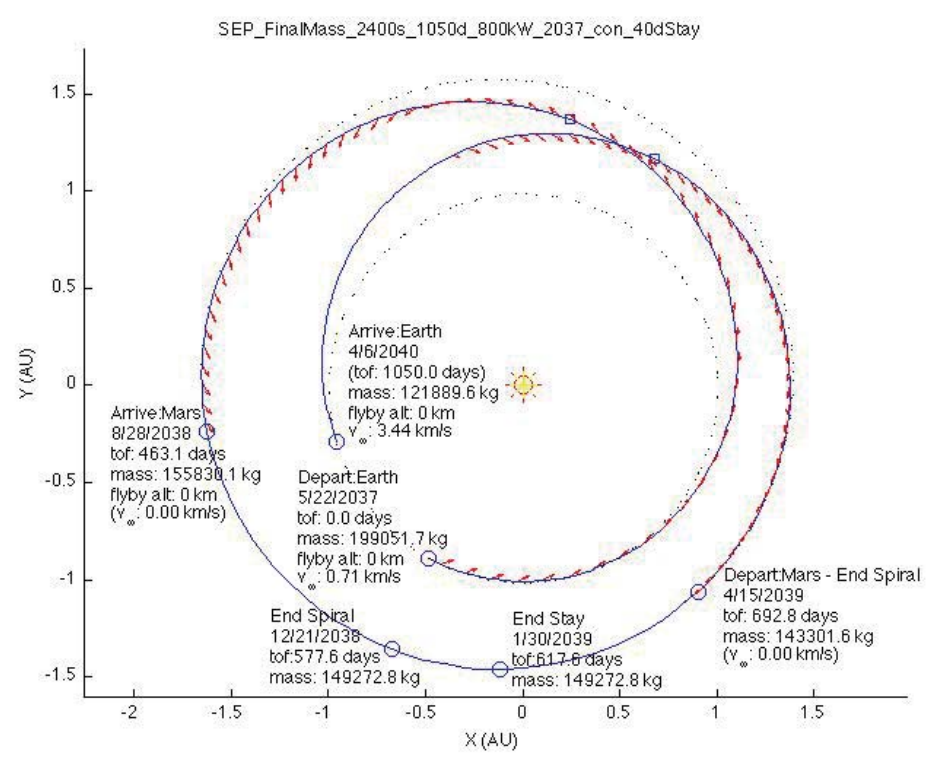

Figure 16. Ecliptic View of $2037800 \mathrm{~kW}$ All-SEP Reference Trajectory

The all-SEP vehicle spends approximately 190 days spiraling in to and out of the 24-hr Mars orbit. With a fixed heliocentric trip time of 1050 days, this combined 190 days of spiraling in the Mars gravity well significantly cuts into the potential stay time at Mars. This is a large reason why the stay time at Mars is much lower compared to the SEP-Chem baseline case.

In order to achieve a 300 day stay at Mars it was necessary to increase the power level. Specifically, all-SEP missions with constrained 300 day stay times were analyzed for power levels of $1400 \mathrm{~kW}$ and 1500 $k W$.

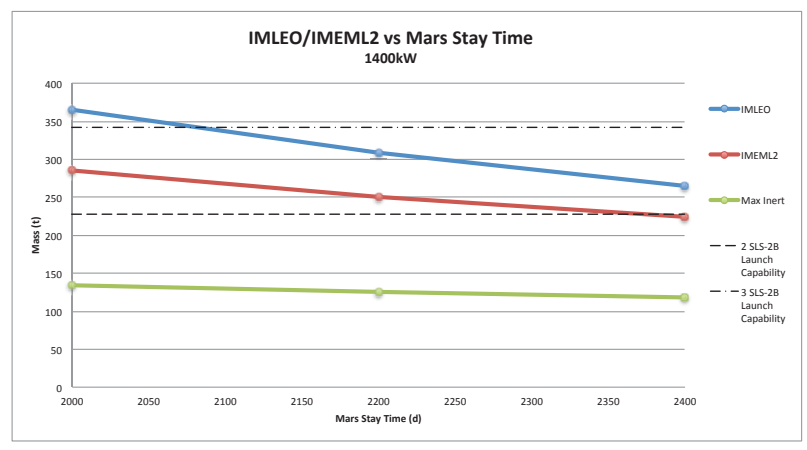

(a) $1400 \mathrm{~kW}$

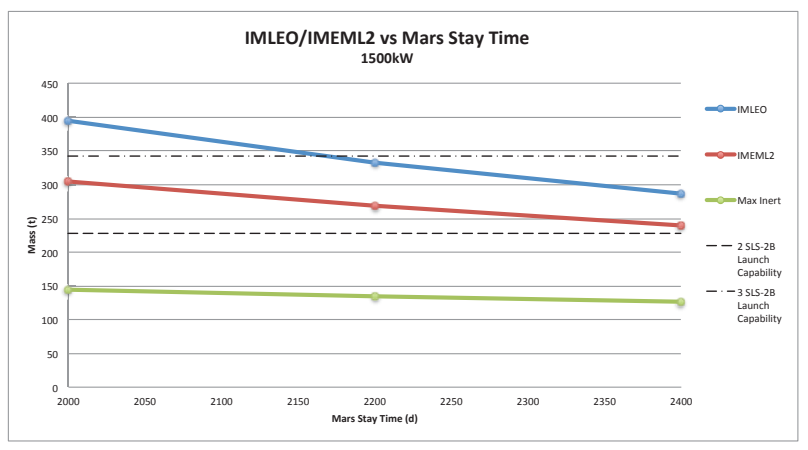

(b) $1500 \mathrm{~kW}$

Figure 17. Mass vs. Stay Time for High Power All-SEP Mission

From Figure 17, a 300 day stay at Mars appears to be possible at power levels of $1400 \mathrm{~kW}$ to $1500 \mathrm{~kW}$ operating at $I_{s p}$ 's of $2000 \mathrm{~s}$ to $2400 \mathrm{~s}$. One exception is that for the $1400 \mathrm{~kW}$ system operating at an $I_{s p}$ of $2400 \mathrm{~s}$ the estimated inert mass for the vehicle using the sizing assumptions from Section A exceeds the maximum allowable inert mass. Although a 300 day stay is achievable with an all-SEP vehicle this option results in an IMLEO substantially above the capability of two SLS-2B launches. Additionally, these options will require power and SEP systems almost twice the size of the the $800 \mathrm{~kW}$ SEP-Chem vehicle capable of performing the same mission. 


\section{Conclusions}

The SEP-Chem concept could be considered a "Poor-Man's Nuclear Bimodal", utilizing SEP in place of NEP and a storable chemical system in place of NTR. This analysis supported a COMPASS design of a SEPChem vehicle which showed that a power limited vehicle (less than $1 M W$ ) has the ability to perform piloted Mars missions if a relatively small storable bipropellant system is integrated. This vehicle can accomplish an Earth-Mars roundtrip mission in approximately 1050 days with a 300 day orbital Mars stay in an elliptical 1-sol orbit. Utilizing SEP in human architectures can be challenging due to the long thrusting time required in Earth and Mars gravity wells. However, by having the SEP-Chem vehicle autonomously spiral out to an EM-L2 Halo orbit where it will rendezvous with the crew prior to departure and performing MOI and TEI with a chemical system, the crewed interplanetary transit times can be kept to roughly 750 days compared to 450-550 days for an NTR or all-chemical conjunction-class mission. However, this additional time is offset by the required number of SLS-2B launches: 3-7 for NTR or all-chemical and just 2 for SEP-Chem. As shown, for lower power vehicles, the chemical system is essential for extending the stay time in Mars orbit. Although less efficient than an SEP capture and departure at Mars, the chemical system eliminates the need for the long spiral-in and spiral-out phases and in doing so extends the length of the stay by roughly 190 days.

The benefits of a SEP-Chem architecture for piloted Earth-Mars orbital stay missions is easily discernible when it is compared with similar architectures utilizing various other propulsive technologies. The crew portion of this architecture can be fully accomplished with two SLS-2B launches for the SEP-Chem modules plus two ATV launches for delivery of additional propellant as well as a single SLS-1B launch to deliver six crew members aboard the MPCV to the EM-L2 Halo orbit. Although during the COMPASS design session it was impossible to explore the design space in it's entirety or to identify the impact of every possible technology trade, a sufficient number of architecture and technology options were explored that these results can be considered representative of an SEP-Chem mission and vehicle.

\section{Acknowledgements}

The authors would like to thank Derrick Cheston and Gary Kelm (NASA/GRC) for their encouragement and interest in this work, as well as, Bret Drake, Carolyn Mercer and Tim Smith (NASA/GRC) for initiating and participating in this COMPASS design. The authors would also like to thank the members of the COMPASS team for their outstanding technical expertise and hard work that went into completing this study.

\section{References}

\footnotetext{
${ }^{1}$ Mercer, C., Oleson, S., Drake, B., A Combined Solar Electric and Storable Chemical Propulsion Vehicle for Piloted Mars Missions, AIAA, 2013. 2009.

${ }^{2}$ Mars Architecture Steering Group., Human Exploration of Mars Design Reference Architecture 5.0, NASA-SP-2009-566,

${ }^{3}$ Oleson, S., McGuire, M., COMPASS Final Report: Piloted Mars Combined SEP/Chemical Design Study, Version 9, 2012.

${ }^{4}$ Oleson, S., Piloted Mars Combined SEP-Chem. Conjunction Piloted and Cargo Designs, Powerpoint Presentation, December 142012 .

${ }^{5}$ Ocampo, C., Senent, J., THE DESIGN AND DEVELOPMENT OF COPERNICUS: A COMPREHENSIVE TRAJECTORY DESIGN AND OPTIMIZATION SYSTEM, IAC-06-C1.4.04, 2006.

${ }^{6}$ Kowalkowski, T., Rinderle, E., Landau, D., Mission-Analysis Low-Thrust Optimization (MALTO) Users' Manual, California Institute of Technology, 2008.

${ }^{7}$ Geffre, J., Jermstad, W., Orion Capabilities for Waypoint Missions, 11 January 2012.

${ }^{8}$ NASA, HAT Cycle C: Mars Transit Habitat and Mars Surface Habitat Modeling, Internal document, August 182011.
} 